\title{
Mechanisms of ischemic stroke in patients with intracranial atherosclerosis: A high-resolution magnetic resonance imaging study
}

\author{
TIANLI GAO ${ }^{1}$, WEI YU ${ }^{2}$ and CHUNJIE LIU ${ }^{1}$ \\ Departments of ${ }^{1}$ Neurology and ${ }^{2}$ Radiology, Beijing Anzhen Hospital, Capital Medical University, Beijing 100029, P.R. China
}

Received September 25, 2013; Accepted February 14, 2014

DOI: $10.3892 /$ etm.2014.1600

\begin{abstract}
High-resolution magnetic resonance imaging (HRMRI) has a unique ability to provide an evaluation of the intracranial artery wall. This study aimed to investigate the possible mechanisms of ischemic stroke in patients with intracranial atherosclerosis using HRMRI. HRMRI was performed on 55 patients (38 male and 17 female) with acute cerebral infarction to investigate the lumen-intruding plaque at the stenotic portion of the middle cerebral artery (MCA) and basilar artery (BA) and to attempt to identify the mechanisms of stroke. Penetrating artery disease (PAD) was diagnosed in 20 patients $(36 \%)$ and large-artery atherosclerosis (LAA) was diagnosed in 35 patients, including 19 with parent artery plaques occluding a penetrating artery (POPA; 35\%) and 16 with artery-to-artery embolisms (29\%). Patients with PAD had a higher frequency of hypertension compared with that of the patients with LAA ( 80 versus $29 \%$; $\mathrm{P}<0.001$ ), and patients with LAA had a higher frequency of diabetes compared with that of the patients with PAD (40\% versus $15 \%$; $\mathrm{P}=0.054)$. Magnetic resonance angiography revealed mild to moderate stenosis in the patients with POPA, while border zone infarction and artery-to-artery embolism occurred in the majority of the patients with severe stenosis or occlusion of the MCA and BA. HRMRI has the ability to identify the mechanisms of intracranial atherosclerotic ischemic stroke through the detection of luminal plaques.
\end{abstract}

\section{Introduction}

Atherosclerosis of the intracranial arteries is frequent and may account for almost one-third of ischemic strokes in the Chinese population $(1,2)$. The underlying mechanisms of

Correspondence to: Dr Tianli Gao, Department of Neurology, Beijing Anzhen Hospital, Capital Medical University, 2 Anzhen Road, Chaoyang, Beijing 100029, P.R. China

E-mail: azyy_gtl@126.com

Key words: high-resolution magnetic resonance imaging, cerebral artery stenosis, ischemic stroke, intracranial atherosclerosis cerebral infarction include artery-to-artery embolism, hemodynamic compromise, local branch occlusion or a combination of those conditions (3). Clique histological study of the middle cerebral artery (MCA) has demonstrated that luminal stenosis is frequently caused by ruptured vulnerable plaques, which are characterized by their specific morphology and composition, which comprises a large lipid/necrotic core covered by a thin fibrous cap infiltrated by macrophages and intraplaque hemorrhage (4). Each clique corresponds to a pair of neighboring pixels, and the clique potential is designed to favor similar classes in neighboring pixels. Progression and a greater extent of intracranial atherosclerosis imply a higher risk of recurrence (5).

High-resolution magnetic resonance imaging (HRMRI) has a unique ability to provide information on plaque composition comparable to that obtained by histology (6). Several studies have confirmed the feasibility of using HRMRI to evaluate the intracranial artery wall and have identified the presence of arterial plaques using HRMRI, in cases where magnetic resonance angiography (MRA) showed no lumen abnormality (7-9). In the present study, HRMRI was used to identify the ischemic stroke subtypes of patients with intracranial atherosclerosis and to investigate the possible mechanisms.

\section{Material and methods}

Study population. A single-center, prospective trial was conducted in the Neurology Department of Beijing Anzhen Hospital, Capital Medical University (Beijing, China) between January 2010 and January 2013. A total of 55 patients with acute cerebral infarction were screened and were subsequently tested for $\geq 50 \% \mathrm{MCA}$ and basilar artery (BA) stenosis by cranial MRI and MRA. The ischemic stroke in these patients was presumed to be caused by atherosclerotic disease. Inclusion criteria were as follows: i) unilateral middle cerebral artery stenosis $(\geq 70 \%)$ or occlusion due to atherosclerosis were observed while no ipsilateral internal carotid artery stenosis or occlusion could be found; ii) there was infarcted focus within corresponding stenosed artery on MRI. iii) Patients must have at least one of the risk factors for atherosclerosis, including hypertension, diabetes, hyperlipidemia, homocysteine and smoking. Exclusion criteria included: i) patients with ipsilateral internal carotid artery stenosis or 
Table I. Baseline characteristics of patients with PAD and LAA.

\begin{tabular}{lccr}
\hline Characteristic & PAD $(\mathrm{n}=20)$ & LAA $(\mathrm{n}=35)$ & P-value \\
\hline Mean age (years; SD, range) & $62(14.5,35-81)$ & $58(12.1,47-75)$ & 0.569 \\
Gender (male/female) & $13 / 7$ & $25 / 10$ & 0.619 \\
Hypertension & $16(80 \%)$ & $10(29 \%)$ & $<0.001$ \\
DM & $3(15 \%)$ & $14(40 \%)$ & 0.054 \\
Hyperlipidemia & $4(20 \%)$ & $13(37 \%)$ & 0.792 \\
History of smoking & $8(40 \%)$ & $9(26 \%)$ & 0.834 \\
History of CAD & $5(25 \%)$ & $7(20 \%)$ & 0.953 \\
History of CVD & $3(15 \%)$ & 0.644 \\
\hline
\end{tabular}

PAD, penetrating artery disease; LAA, large-artery atherosclerosis; DM, diabetes mellitus; CAD, coronary artery disease; CVD, cerebrovascular disease.

occlusion. ii) Non-atherosclerotic cerebral artery stenosis, such as fibromuscular dysplasia, arteritis and dissecting aneurysm. iii) Patients suspected to have symptoms of cardiogenic embolism, including recent myocardial infarction, atrial fibrillation with or without mural thrombus, mitral stenosis or prosthetic valve, dilated cardiomyopathy, sick sinus syndrome, acute bacterial endocarditis and patent foramen ovale. Written informed consent was obtained from all patients. The Ethical Committee of Beijing Anzhen Hospital approved this study (Beijing, China).

Clinical assessment. All patients underwent a detailed medical history assessment and a physical examination at baseline that included routine blood biochemistry tests, coagulation testing, transthoracic or transesophageal echocardiography, Holter electrocardiography, transcranial Doppler sonography, carotid ultrasound, and computed tomography, MRI and MRA of the brain. Data collected from patients included the following baseline characteristics: Age, gender, vascular risk factors, such as hypertension or history of hypertension or its complications, history of diabetes mellitus (DM) or currently diagnosed DM, hyperlipidemia, history of smoking, previous coronary artery disease and previous cerebrovascular disease.

Ischemic stroke subclassification. According to the Chinese ischemic stroke subclassification (10), the patients were grouped into two mechanism-based categories: Penetrating artery disease (PAD; no evidence of atherosclerotic plaques or any degree of stenosis in the parent artery) and large-artery atherosclerosis (LAA; plaques in the parent artery occluding a penetrating artery, artery-to-artery embolism or hypoperfusion/impaired emboli clearance).

MRI protocol and review. All patients were imaged at the Beijing Anzhen Hospital using a Magnetom Verio 3T MRI scanner (Siemens AG, Erlangen, Germany) and an eight-channel brain-array coil. A standardized protocol was used to perform conventional brain T1- and T2-weighted MRI and three-dimensional (3D) time-of-flight (TOF)-MRA. 3D TOF-MRA data were obtained using an axial plane with a repetition time (TR)/echo time (TE) of $21 \mathrm{msec} / 3.6 \mathrm{msec}$; flip angle of $18^{\circ}$; field-of-view (FOV) of 220x220 mm; slice thickness of $0.5 \mathrm{~mm}$; and a matrix size of $320 \times 380$ pixels. The TOF-MRA scan time was $4 \mathrm{~min}$. MRA data were reconstructed using a dedicated online post-processing tool [multiplanner reconstruction (MPR), maximum intensity (MIP), volume rendering (VR)] to determine the blood vessel architecture.

HRMRI data were acquired from the patients with MCA and BA steno-occlusive lesions along the short axes of the stenotic segments on TOF-MRA images. The lesion site for the evaluation of unilateral MCA stenosis was determined by the interpreting neuroradiologist as the ipsilateral MCA in the symptomatic patients and the side of severe MCA stenosis in the asymptomatic patients. T1- and T2-weighted MRI was centered at the stenosis of the MCA-M1 segment, vertebral artery, BA and their confluences. The MRI parameters were: T1-weighted, double inversion recovery, black blood, two-dimensional turbo spin echo (TSE), $\mathrm{TR} / \mathrm{TE}=920 \mathrm{msec} / 27 \mathrm{msec}, \mathrm{FOV}=120 \times 120 \mathrm{~mm}$, matrix size $=270 \times 320$ pixels, slice thickness $=2.0 \mathrm{~mm}$ and $2 \mathrm{NEX}$. For the T2-weighted HRMRI scans, the TSE sequence used a TR/TE of 2,350 msec/78 msec, FOV of 120x120 mm, matrix size of 270x320 pixels, slice thickness of $2.0 \mathrm{~mm}$ and $2 \mathrm{NEX}$. The black blood technique with pre-regional saturation pulses of $80-\mathrm{mm}$ thickness to saturate incoming arterial flow was used for the scans. The longitudinal coverage of each artery was $16 \mathrm{~mm}$ (eight slices) for the two types of scans, with a scan time of $\sim 3-4 \mathrm{~min} / \mathrm{scan}$. The total scan time was $\sim 20 \mathrm{~min}$ and the patients remained in the MRI machine for $\sim 30 \mathrm{~min}$.

Statistical analysis. A $\chi^{2}$ test was used to compare frequencies. One-way analysis of variance and Student's t-test were used for normally distributed variables, whereas the Mann-Whitney $\mathrm{U}$ test was used for non-normally distributed variables. All tests of statistical significance were two-sided, with $\mathrm{P}<0.05$ considered to indicate a statistically significant difference. All statistical analyses were performed using SPSS software, version 17.0 (SPSS, Inc., Chicago, IL, USA).

\section{Results}

A total of 55 patients who met the eligibility criteria were recruited to this study. There were 38 males and 17 females with a median age of 58.9 years (standard deviation, 
A
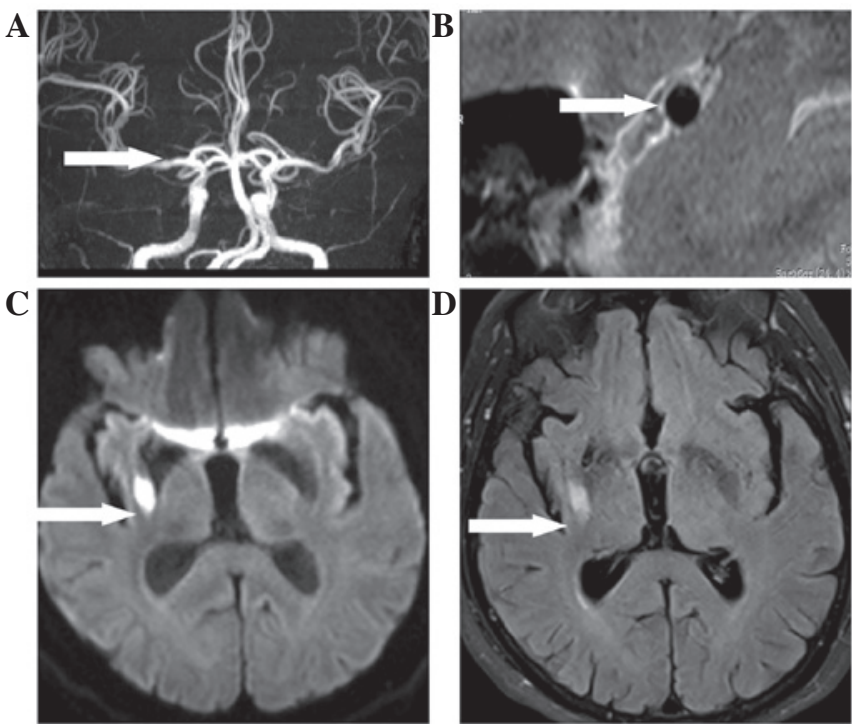

Figure 1. A 72-year-old male presented with left hemiparesis and dysarthria. (A) Right MCA wall MRA indicates mild-grade MCA stenosis (B) T2-weighted HRMRI cross-sectional image of the MCA (M1 segment) reveals a normal circular lumen with no wall thickening. (C) A single small infarction of a penetrating artery is shown using DWI. (D) T2 fluid-attenuated inversion-recovery imaging indicates an infarction lesion located at the same position, corresponding to the territory of a penetrating artery of the right MCA. The arrows represent the position of the lesion. MCA, middle cerebral artery; HRMRI, high-resolution magnetic resonance imaging; DWI, diffusion-weighted imaging.

\pm 13.4 years; range, 35-81 years). Among the 55 patients, 17 had vertebrobasilar stenosis $(31 \%)$ and 38 had MCA stenosis (69\%). PAD was diagnosed in 20 of the patients $(36 \%)$ and LAA was diagnosed in 35 of the patients, which included 19 with a parent artery plaque occluding a penetrating artery (POPA) (35\%) and 16 with artery-to-artery embolism and/or hypoperfusion $(29 \%)$. The baseline characteristics in the two groups were comparable, with the exceptions that the patients with PAD had a higher frequency of hypertension compared with that of the patients with LAA (80 versus 29\%; $\mathrm{P}<0.001$ ) and the patients with LAA had a higher frequency of DM than that of the patients with PAD (40 versus $15 \%$; $\mathrm{P}=0.054$ ) (Table I). POPA occurred more frequently in the patients with mild to moderate artery stenosis $(63 \%$; $\mathrm{P}<0.05)$ than in the patients with severe artery stenosis or occlusion $(37 \%)$. However, the stroke mechanisms of artery-to-artery embolism and/or hypoperfusion were mainly observed in the patients with severe artery stenosis or occlusion (68\%) compared with in the patients with mild to moderate artery stenosis (13.3\%; $\mathrm{P}=0.060)$.

HRMRI enabled the detection of the lumen wall, and the MCA and BA were clearly observed in all cases. The cross-sectional imaging findings of patients who were diagnosed with LAA indicated that the presence of focal arterial wall thickening was consistent with a plaque on the level of the MCA and BA stenotic area on MRA images. The plaque appeared as a crescent-shaped or eccentric thickening surrounding a circular lumen. As shown in Fig. 1, in a 72-year-old male patient, MRA revealed relatively mild stenosis in the MCA-M1 segment, while HRMRI showed no lumen abnormality. A high-intensity infarct lesion revealed by
A
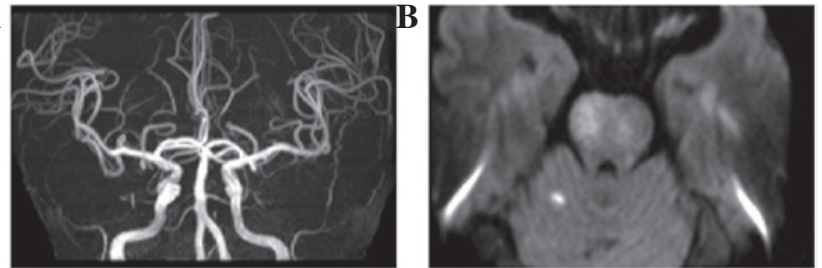

C
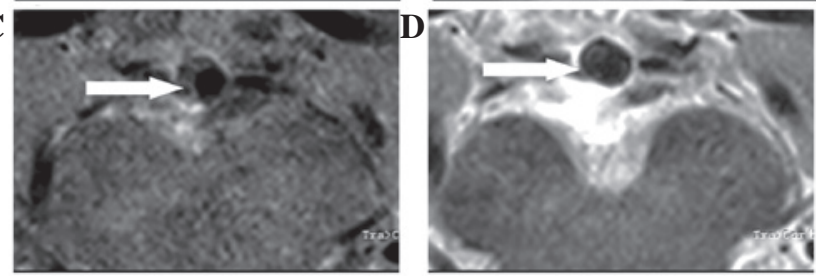

Figure 2. A 60-year-old female presented with an episode of sudden onset left face, arm and leg weakness. (A) MRA revealed an irregular mild BA stenosis close to the pontine paramedian artery. (B) An acute high-intensity infarction was demonstrated on the axial DWI image at the level of the right lateral pontine. (C and D) Cross-sectional T1- and T2-weighted HRMRI at the level of the BA revealed a lipid-rich necrotic eccentric plaque with low or iso-signal density. The arrows represent the position of the lesion. MRA, magnetic resonance angiography; BA, basilar artery; DWI, diffusion-weighted imaging; HRMRI, high-resolution magnetic resonance imaging.
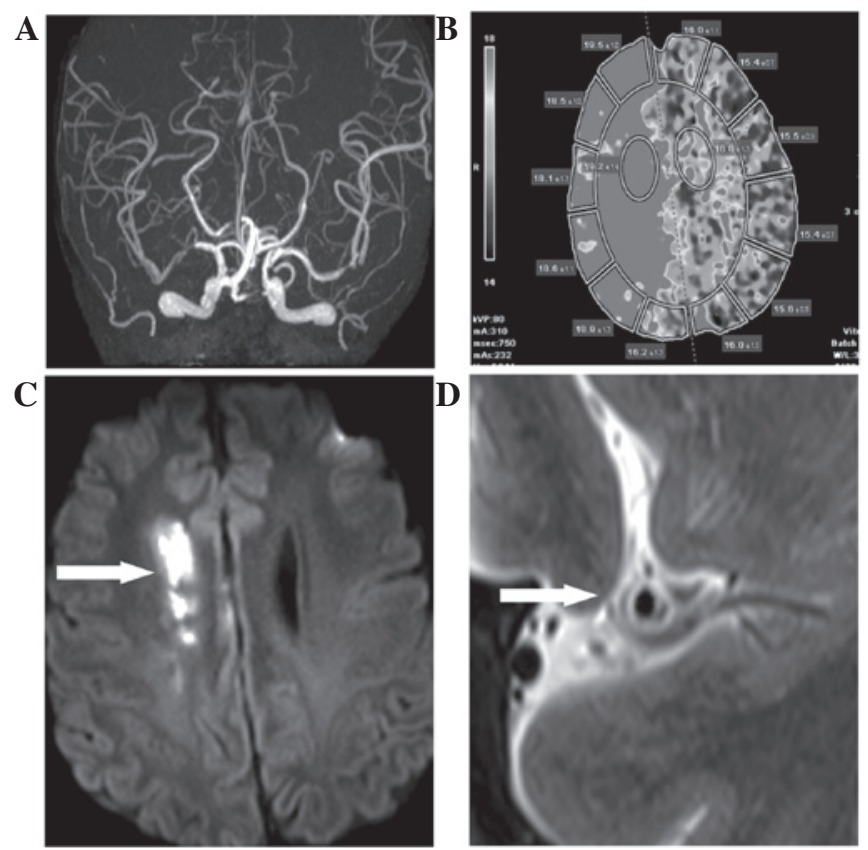

Figure 3. A 71-year-old female patient presented with dysarthria and mild left hemiparesis. (A) MRA from the right proximal MCA (M1 segment) showed a focal severe stenosis. (B) DWI confirmed internal border-zone infarction, which corresponded to the right MCA territory with the underlying mechanism of hypoperfusion and impaired emboli clearance. (C) Computed tomography perfusion imaging also demonstrated relatively low cerebral blood flow, and elevated time-to-peak in a region of the right MCA indicated the potential of hypoperfusion. (D) The T2-weighted HRMRI cross-sectional lumen image showed a hyposignal eccentric core surrounded by a high-signal thickened wall. The arrows represent the position of the lesion. MRA, magnetic resonance angiography; MCA, middle cerebral artery; DWI, diffusion-weighted imaging; HRMRI; high-resolution magnetic resonance imaging.

diffusion-weighted imaging (DWI) was present in the lateral striate arterial territory, indicating that this was a case of the PAD stroke subtype. HRMRI findings of the lumen elucidated 
the underlying mechanisms in which lipohyalinosis or microatheroma may be the etiology for PAD. Furthermore, in a 60 -year-old female patient, MRA showed relatively mild to moderate stenosis in the BA where an eccentric plaque located in the lumen wall was shown to occlude the paramedian pontine arteries by HRMRI. An acute ischemic infarction was verified by DWI (Fig. 2). This was indicated to be a typical case, where the infarction was confined to the territory of a single branch artery or a few penetrating branches that were occluded by the plaques of their parent arteries. Borderzone infarction and artery-to-artery embolism occurred in the most severe stenosis and occlusion of the MCA and BA. Computed tomography perfusion imaging of a 71-year-old female patient demonstrated relatively low cerebral blood flow and elevated time-to-peak in a region of the MCA, indicating the potential mechanism of hypoperfusion (Fig. 3).

\section{Discussion}

Intracranial atherosclerotic disease causes ischemic strokes, and the rates of recurrent vascular ischemic events and vascular mortalities are very high (11). However, intracranial atherosclerosis, which affects cerebral arteries such as the BA and MCA, remains an infradiagnosed and understudied disease (12). The diagnosis of intracranial stenosis is traditionally dependent on conventional angiography and several reliable noninvasive diagnostic methods, including transcranial color-coded duplex sonography, MRA and computed tomography angiography $(13,14)$. Cross-sectional HRMRI is a promising technique for imaging carotid plaques, with a sensitivity of $85 \%$ and a specificity of $92 \%$ for identifying soft plaques (necrotic core or hemorrhage) (15-17). HRMRI has consistently emerged as a potential technique for imaging atherosclerotic plaques in the intracranial arteries (7). This imaging technique may provide information about the histopathological nature of the intracranial atherosclerotic lesion responsible for arterial narrowing (9).

As no underlying cause is found in $>30 \%$ of stroke cases, HRMRI detection of intracranial atherosclerotic lesions may have significant clinical implications (3). Detection of the vessel wall of arteries such as the MCA and BA may improve the ability to identify advanced but unrecognized intracranial atherosclerotic disease (18). In the present study, the features of the MCA and BA luminal wall in patients who had suffered cerebral infarction were explored. Recognizing the characteristics of LAA and PAD may contribute to improved risk stratification and allow aggressive interventions to be targeted at patients with plaques that are prone to rupture (19-21). A previous study of carotid artery plaques has demonstrated a significant correlation between plaque characteristics identified by HRMRI and subsequent stroke patterns (19). In the present study, HRMRI clearly confirmed the presence of a reduced arterial lumen associated with a focal wall thickening and plaques at the level of MCA and BA stenosis, suggesting that the HRMRI technique was useful in determining the etiology of PAD and POPA.

In a comparison of the patients with PAD and those with LAA, it was observed that patients with PAD had a higher frequency of hypertension whereas patients with LAA had a higher frequency of DM. Aging and chronic hypertension are risk factors for large-artery atherosclerosis (22). Such changes include replacement of the smooth muscle cells by fibro-hyaline material with thickening of the wall and narrowing of the vascular lumen. Arteriolosclerosis may be one of the reasons that the blood supply to the white matter is altered, and this vascular change may lead to localized ischemic areas of necrosis and cavitations (14). Previous studies have shown a high frequency of intracranial stenosis in diabetic Caucasian patients, an independent association of type II DM to a greater extent of intracranial LAA, and a significantly higher number of diseased vessels in diabetic patients compared with that in nondiabetic patients $(13,23)$. The association between diabetes and more diffuse and advanced intracranial atherosclerosis is unclear. Consequently, among the traditional vascular risk factors, diabetes appears to play a preeminent role in intracranial macroangiopathy in the Chinese population (24). Additionally, the presence of intracranial LAA disease contributes to a poorer outcome for patients with LAA disease, which may be stratified as very high risk in secondary prevention (25).

In conclusion, stroke patterns of intracranial atherosclerotic arteries are complicated and mainly include LAA and PAD. HRMRI has the ability to identify the mechanisms behind intracranial atherosclerotic ischemic stroke by showing the luminal wall. It may also provide a useful tool in risk stratification and the selection of candidates for invasive therapies.

\section{References}

1. Pu Y, Liu L, Wang Y, et al; Chinese IntraCranial AtheroSclerosis (CICAS) Study Group: Geographic and sex difference in the distribution of intracranial atherosclerosis in China. Stroke 44: 2109-2114, 2013.

2. Rincon F, Sacco RL, Kranwinkel G, et al: Incidence and risk factors of intracranial atherosclerotic stroke: the Northern Manhattan Stroke Study. Cerebrovasc Dis 28: 65-71, 2009.

3. Shi MC, Wang SC, Zhou HW, et al: Compensatory remodeling in symptomatic middle cerebral artery atherosclerotic stenosis: a high-resolution MRI and microemboli monitoring study. Neurol Res 34: 153-158, 2012.

4. Naghavi M, Libby P, Falk E, et al: From vulnerable plaque to vulnerable patient: a call for new definitions and risk assessment strategies: Part I. Circulation 108: 1664-1672, 2003.

5. Xu WH, Li ML, Gao S, et al: In vivo high-resolution MR imaging of symptomatic and asymptomatic middle cerebral artery atherosclerotic stenosis. Atherosclerosis 212: 507-511, 2010.

6. Ma N, Jiang WJ, Lou X, et al: Arterial remodeling of advanced basilar atherosclerosis: a 3-tesla MRI study. Neurology 75: 253-258, 2010.

7. Bodle JD, Feldmann E, Swartz RH, Rumboldt Z, Brown T and Turan TN: High-resolution magnetic resonance imaging: an emerging tool for evaluating intracranial arterial disease. Stroke 44: 287-292, 2013.

8. Niizuma K, Shimizu H, Takada S and Tominaga T: Middle cerebral artery plaque imaging using 3-Tesla high-resolution MRI. J Clin Neurosci 15: 1137-1141, 2008.

9. Ryu CW, Jahng GH, Kim EJ, Choi WS and Yang DM: High resolution wall and lumen MRI of the middle cerebral arteries at 3 tesla. Cerebrovasc Dis 27: 433-442, 2009.

10. Gao S, Wang YJ, Xu AD, et al: Chinese ischemic stroke subclassification. Front Neurol 2: 6, 2011.

11. Turan TN, Maidan L, Cotsonis G, et al; Warfarin-Aspirin Symptomatic Intracranial Disease Investigators: Failure of antithrombotic therapy and risk of stroke in patients with symptomatic intracranial stenosis. Stroke 40: 505-509, 2009.

12. Kim JM, Jung KH, Sohn CH, Moon J, Han MH and Roh JK: Middle cerebral artery plaque and prediction of the infarction pattern. Arch Neurol 69: 1470-1475, 2012. 
13. Arenillas JF and Alvarez-Sabin J: Basic mechanisms in intracranial large-artery atherosclerosis: advances and challenges. Cerebrovasc Dis 20 (Suppl 2): 75-83, 2005.

14. Yazdani SK, Vorpahl M, Ladich E and Virmani R: Pathology and vulnerability of atherosclerotic plaque: identification, treatment options, and individual patient differences for prevention of stroke. Curr Treat Options Cardiovasc Med 12: 297-314, 2010.

15. Cai JM, Hatsukami TS, Ferguson MS, Small R, Polissar NL and Yuan C: Classification of human carotid atherosclerotic lesions with in vivo multicontrast magnetic resonance imaging. Circulation 106: 1368-1373, 2002.

16. Kampschulte A, Ferguson MS, Kerwin WS, et al: Differentiation of intraplaque versus juxtaluminal hemorrhage/thrombus in advanced human carotid atherosclerotic lesions by in vivo magnetic resonance imaging. Circulation 110: 3239-3244, 2004.

17. Larose E, Yeghiazarians Y, Libby P, et al: Characterization of human atherosclerotic plaques by intravascular magnetic resonance imaging. Circulation 112: 2324-2331, 2005.

18. Lam WW, Wong KS, So NM, Yeung TK and Gao S: Plaque volume measurement by magnetic resonance imaging as an index of remodeling of middle cerebral artery: correlation with transcranial color Doppler and magnetic resonance angiography. Cerebrovasc Dis 17: 166-169, 2004.
19. Gao T, Zhang Z, Yu W, Zhang Z and Wang Y: Atherosclerotic carotid vulnerable plaque and subsequent stroke: a high-resolution MRI study. Cerebrovasc Dis 27: 345-352, 2009.

20. Mono ML, Karameshev A, Slotboom J, et al: Plaque characteristics of asymptomatic carotid stenosis and risk of stroke. Cerebrovasc Dis 34: 343-350, 2012.

21. Lindsay AC, Biasiolli L, Lee JM, et al: Plaque features associated with increased cerebral infarction after minor stroke and TIA: a prospective, case-control, 3-T carotid artery MR imaging study. JACC Cardiovasc Imaging 5: 388-396, 2012.

22. Pantoni L and Garcia JH: Pathogenesis of leukoaraiosis: a review. Stroke 28: 652-659, 1997.

23. Arenillas JF, Molina CA, Chacón P, et al: High lipoprotein (a), diabetes, and the extent of symptomatic intracranial atherosclerosis. Neurology 63: 27-32, 2004.

24. Thomas GN, Lin JW, Lam WW, et al: Albuminuria is a marker of increasing intracranial and extracranial vascular involvement in Type 2 diabetic Chinese patients. Diabetologia 47: 1528-1534, 2004.

25. Kwan MW, MakW, Cheung RT and HoSL: Ischemic stroke related to intracranial branch atheromatous disease and comparison with large and small artery diseases. J Neurol Sci 303: 80-84, 2011. 